\title{
Association of Hemoglobin Levels with Stroke Mortality in Patients of Type 2 Diabetes Mellitus
}

\author{
Dr. Shaheen B Shaikh ${ }^{1}$, Dr. Ismail HM², Miss Roshni ${ }^{3}$, Dr. Shaheena Yassir ${ }^{4 *}$, Dr. Saiqa R ${ }^{4}$, Dr. Sarfaraz Shaikh \\ ${ }^{1}$ Associate Professor, Department of Biochemistry, Yenepoya Medical College Hospital, Mangaluru, Karnataka, India \\ ${ }^{2}$ Associate Professor, Department of Critical Care Medicine, Yenepoya Medical College Hospital, Mangaluru, Karnataka, India \\ ${ }^{3}$ Tutor, Department of Biochemistry, Yenepoya Medical College Hospital, Mangaluru, Karnataka, India \\ ${ }^{4}$ Assistant Professor, Department of Biochemistry, Yenepoya Medical College Hospital, Mangaluru, Karnataka, India \\ ${ }^{5}$ Consultant, Department of Emergency Medicine, D M WIMS Medical College, Wayanad, Kerala India
}

DOI: $10.36348 / \mathrm{sijb} .2020 . \mathrm{v} 03 \mathrm{i} 12.002$

| Received: 26.11.2020 | Accepted: 09.12.2020 | Published: 13.12.2020

*Corresponding author: Dr. Shaheena Yassir

\section{Abstract}

Background: In stroke scenario, decreased hemoglobin levels have the potential to worsen brain ischemia; however, the association between the levels of hemoglobin with stroke mortality is not well understood. Aim: The study investigated the association of hemoglobin levels with 28 days mortality rate after ischemic stroke (IS). Methods: This was a retrospective study, where data of 74 patients admitted to ICU was collected with a diagnosis of stroke .The data were analyzed in the terms of demographic details, plasma glucose, HbA1c and hemoglobin levels. Results: Among 74 analyzed patients, $56.70 \%$ were men and $43.30 \%$ were females. In our study $32.4 \%$ of stroke patients had anemia. Out of $45.9 \%$ non survivors, $21.6 \%$ stroke patients had hemoglobin level less than $11 \mathrm{mg} / \mathrm{dl}$ and $24.30 \%$ stroke patients had hemoglobin level more than $11 \mathrm{mg} / \mathrm{dl}$. Out of $54.1 \%$ survivors , $10.8 . \%$ and $43.20 \%$ stroke patients had hemoglobin level less than $11 \mathrm{mg} / \mathrm{dl}$ and more than $11 \mathrm{mg} / \mathrm{dl}$, respectively .The data was statistically significant. Conclusion: In conclusion, we showed that a significant proportion of stroke patients have low hemoglobin at the time of stroke onset and this is associated with increased mortality up to 28 days. Targeted interventions in this patient population may improve outcomes and require further evaluation.

Keywords: Pulse pressure, Hemoglobin, Glucose, stroke, Brain ischemia, HbA1c.

Copyright (C) 2020 The Author(s): This is an open-access article distributed under the terms of the Creative Commons Attribution 4.0 International License (CC BY-NC 4.0) which permits unrestricted use, distribution, and reproduction in any medium for non-commercial use provided the original author and source are credited.

\section{INTRODUCTION}

Anemia is an exacerbating factor in ischemic stroke patients. The hemoglobin level and anemia has an impact on stroke mortality, and this remains controversial [1]. Studies based on hospital reported prevalence of anemia in stroke patients is upto 30\% [2]. Despite many observational studies having reported an adverse impact of admission low hemoglobin on poststroke mortality outcome [3], the relationship between the two is not well comprehended.

Stroke is an important cause for morbidity and long-term disability. Studies carried to decipher the relation between anemia and mortality in patients of stroke showed controversial outcomes [4]. Studies show highly conflicting results, with some studies showing no significant association [5] and some showing significance [6]. To address the current uncertainties in relationship between stroke and hemoglobin.we aimed to investigate the association of hemoglobin levels with 28 days mortality after Ischemic Stroke (IS).

\section{MATERIALS AND METHODS}

A retrospective study was conducted from January 2019 to December 2019 in the department of biochemistry and Critical Care Medicine, tertiary care hospital. A total of 74 diabetic patients of ischemic stroke admitted in MICU were enrolled in this study. Clearance from institutional ethical committee was obtained and data was collected from all diabetic patients admitted to MICU and the case details were retrospectively retrieved from MRD section. and analyzed for patients which includes demographic details, h/o arterial hypertension (AH), congestive heart failure (CHF), presence of diabetes mellitus (DM), atrial fibrillation $(\mathrm{AF})$, pulse rate $(\mathrm{PR})$, mean arterial blood pressure (MAP), pulse pressure (PP), plasma glucose and hemoglobin levels. Age, sex, duration of ICU stay, duration of DM, other co-morbidities, medication history was recorded for all patients. All patients' records were traced up to 28 days post stroke. 
Other Risk factors like smoking, alcohol and hypertension history were also emphasized. Mortality accounting up to 28 days was taken into consideration; Data was collected \& entered in the hospital based database.

\section{STATISTICAL ANALYSIS}

The data was entered in the excel spread sheet. The descriptive analytical statistics were evaluated statistically with IBM SPSS Statistics for Windows, Version 23.0, IBM Corp, and Chicago, IL. Student's t test, Fisher's exact test and Pearson Chi Square test were used to compare variables as appropriate. Unpaired $\mathrm{T}$ test was used to test the significant difference between Hemoglobin levels with mortality. P value less than 0.05 was considered as statistically significant.

\section{RESULTS}

In our study, a total of 74 diabetic patients, medical records were analyzed retrospectively to investigate association of hemoglobin levels with 28 days mortality in patients admitted with acute ischemic stroke.

Among 74 analyzed patients, $42(56.70 \%)$ were men and $32(43.30 \%)$ were females. The mean age of our study population was 61-70 years. The risk factors for stroke are arterial hypertension in 24 $(30.00 \%)$ patients, congestive heart failure in 34 $(42.50 \%)$ patients and atrial fibrillation in $24(17.50 \%)$ patients.

The clinical data of study population with stroke among survivors and non survivors along with $\mathrm{p}$ value are shown in table 1 . Out of 74 analyzed patients, a total of 36 patients expired during 28 days period of time following stroke (Table 1).

Table-1: Clinical data of study population with stroke

\begin{tabular}{|c|c|c|c|}
\hline Parameters & Survivor $(\mathbf{n}=\mathbf{3 8})$ & Non Survivor( $n=36)$ & P Value \\
\hline Mean Age (years) & $62.26 \pm 3.74$ & $64.85 \pm 4.16$ & $0.000^{*}$ \\
\hline Male $(\mathrm{n}=42)$ & $26(54.16 \%)$ & $16(45.84 \%)$ & NS \\
\hline Females $(n=32)$ & $12(37.50 \%)$ & $20(62.50 \%)$ & NS \\
\hline Glucose (mg /dl ) & $136 \pm 48$ & $139 \pm 59$ & $<0.001 *$ \\
\hline Arterial Hypertension $(n=24)$ & $10(41.66 \%)$ & $14(58.33 \%)$ & 0.05 \\
\hline Congestive Heart Failure $(n=34)$ & $16(47.05 \%)$ & $18(52.94 \%)$ & NS \\
\hline Arterial Fibrillation $(n=24)$ & $4(14.28 \%)$ & $20(85.71 \%)$ & $0.02 *$ \\
\hline Pulse rate (bpm) & $87 \pm 16$ & $91 \pm 13$ & NS \\
\hline Mean arterial pressure $(\mathrm{mm} \mathrm{Hg})$ & $121 \pm 24$ & $129 \pm 19$ & 0.05 \\
\hline $\begin{array}{l}\text { Pulse pressure } \\
(\mathrm{mm} \mathrm{Hg})\end{array}$ & $62 \pm 26$ & $67 \pm 21$ & $<0.001 *$ \\
\hline
\end{tabular}

$* \mathrm{P}<0.05$ is considered as statistically significant.

In our study $32.4 \%$ of stroke patients had anemia with male $(16.2 \%)$ and in females $(16.2 \%)$ with $\mathrm{p}$ value of $>0.05$, shown in Table 2 . The values were statistically significant. Our study showed that non- survivors had higher blood sugar values compared to those of survivors thus showing a significant relationship between blood sugar value \& mortality rate.

Table-2: Baseline Characteristics of Patients with and without Anemia

\begin{tabular}{|l|c|c|l|}
\hline & Patients without Anemia & Patients with Anemia & \multicolumn{1}{|c|}{ P Value } \\
\hline Patients & $(67.6 \%)$ & $24(32.4 \%)$ & $<0.001^{*}$ \\
\hline Males & $30(40.5 \%)$ & $12(16.2 \%)$ & $<0.01^{*}$ \\
\hline Females & $20(27.0 \%)$ & $12(16.2 \%)$ & $<0.001^{*}$ \\
\hline Age (years) & $61.2 \pm 14.18$ & $65.17 \pm 9.62$ & $>0.05$ \\
\hline
\end{tabular}

Out of $74(45.9 \%)$ non survivors, $32(21.6 \%)$ stroke patients had hemoglobin level less than $11 \mathrm{mg} / \mathrm{dl}$ and $36(24.30 \%)$ stroke patients had hemoglobin level more than $11 \mathrm{mg} / \mathrm{dl}$. Out of $40(54.1 \%)$ survivors , 8
$(10.8 . \%)$ stroke patients had hemoglobin level less than $11 \mathrm{mg} / \mathrm{dl}$ and $32(43.20 \%)$ stroke patients had hemoglobin level more than $11 \mathrm{mg} / \mathrm{dl}$ shown in Table 3.

Table-3: Association between hemoglobin and 28 days mortality

\begin{tabular}{|l|l|l|c|}
\hline 28 Days Mortality & Hb $<\mathbf{1 1}$ & Hb $>\mathbf{1 1}$ & Total \\
\hline Non Survivor & 16 & 18 & 34 \\
& $(21.6 \%)$ & $(24.3 \%)$ & $(45.9 \%)$ \\
\hline Survivor & 8 & 32 & 40 \\
& $(10.8 \%)$ & $(43.2 \%)$ & $(54.1 \%)$ \\
\hline Total & 24 & 50 & 74 \\
& $(32.4 \%)$ & $(67.6 \%)$ & $(100.0 \%)$ \\
\hline
\end{tabular}


These data was statistically significant. But 28 days mortality was high in patients with hemoglobin levels less than $11 \mathrm{mg} / \mathrm{dl}$. The association between hemoglobin and 28 days is graphically represented in Fig 1. High 28 days mortality rate was seen when hemoglobin levels were less than $11 \mathrm{mg} / \mathrm{dl} \&$ low mortality rate when hemoglobin levels were more than $11 \mathrm{mg} / \mathrm{dl}$.

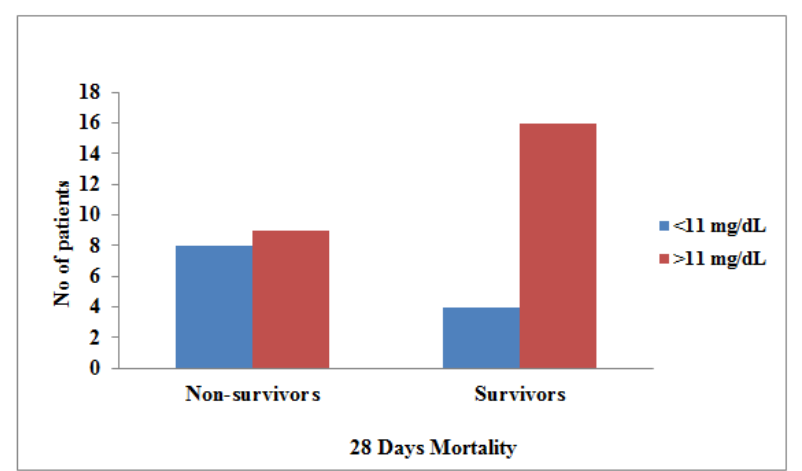

Fig-1: Association between hemoglobin and 28 days Mortality

\section{DISSCUSION}

We investigated the relationship between anemia, the levels of hemoglobin and 28 days mortality in patients with acute stroke. An increased mortality of up to 28 days was found in a good number of stroke patients with anemia at onset of stroke. We observed that low hemoglobin on admission was a frequent condition in patients with stroke.

The presence of arterial hypertension, congestive heart failure and atrial fibrillation as well as higher PR, MAP and serum glucose were also associated with significant mortality in patients with 30 day period of post stroke [7, 8]. There are many pathological mechanisms in literature that can explain the relation between anemia and higher risk of mortality in stroke patients.

First, low hemoglobin may increase mortality in stroke due to its association with mediators of inflammation, which increases the synthesis of nitric oxide synthase and CXC chemokine receptor 4 . These have been linked with brain injury $[9,10]$.

Second, anemia because of low oxygencarrying capacity of blood may exaggerate ischemia and hypoxia in the penumbral lesions in ischemic stroke patients [11,12].Third, anemia may lead to hyperdynamic circulation and activate an provocative response that leads to formation of thrombus, like that of atherosclerosis [13,14].

Fourth, an increase in cerebral blood flow causing turbulence triggers the movement of an existing thrombus and leading to the process thromboembolism [15].
The association of mortality rate and transfusion in patients with anemia having stroke, has been reported in some observational studies. While some depicting a fall in mortality rate [16], others show no change [17].

The association between low hemoglobin and mortality suggests that, timely interventions may improve outcomes. Raphae S. Barlas et al; Strongly suggests that there is an increased mortality in stroke patients with anemia [4].Bhupesh $\mathrm{P}$ et al; concluded that lower and higher hemoglobin concentrations were associated with a higher risk of incident stroke in women[18]. Kannel et al; examined the association between hemoglobin concentration and incident stroke in the Framingham cohort. They reported an increased risk of stroke with increasing hemoglobin concentration within the reported normal range of hemoglobin [19].

Zilong Hao et al; this study indicates that more than one in four patients with acute ischemic stroke had anemia, and that anemia is an independent predictor of death in patients with acute ischemic stroke [20]. Tanne $\mathrm{D}$ et al. concluded that anemia in both the sexes has similar prevalance in stroke patients and has poor prognosis. In addition, there is a nonlinear association between hemoglobin at the time of admission and mortality. The mortality outcome increases at both high and low hemoglobin levels [21].

Our findings suggest that it might be useful to treat patients with acute stroke with anemia as early as possible to reduce deleterious effects. The beneficial effect of increasing oxygen-carrying capacity by blood transfusion needs counterpoising by acute complications like infection, hemolytic reactions, volume overload, and inflammatory processes activation [22].

Though this result seems promising regarding association between MICU mortality and hemoglobin levels, additional data is required for this particular context. Further investigations are needed to assess the etiology of raised mortality in patients with extremes of hemoglobin concentrations and also to find out whether there is an improvement in long-term outcome after stroke by achieving a target hemoglobin concentrations .

LIMITATION: This is a retrosecptive study; hence the cause cannot be concluded from the observed associations. Small sample sizes and a majority of patients did not report outcomes by stroke. We had no information on the duration or cause of anemia.

\section{CONCLUSION}

To conclude, we found that a good number of stroke patients had low hemoglobin at the onset of stroke. An increased rate of mortality up to 28 days was found in such patients. In such patients, targeted interventions may improve outcomes and require 
further assessment. Further studies are needed to examine the effectiveness of interventions in terms of clinical and financial outcomes in patients in an acute stroke setting. Since low hemoglobin levels are correctable abnormalities in the stroke patients, they should be managed accordingly as a therapeutic target so as to improve the outcome in hospital admitted patients \& reduce the mortality.

\section{REFERENCES}

1. Sharma, K., Johnson, K. (2018). Hemoglobin concentration does not impact 3-month outcome following acute ischemic Stroke: BMC Neurology, June; 18:78-81.

2. Sico, J., Concato, J. (2013). Anemia is associated with poor outcomes in patients with less severe ischemic stroke. J Stroke Cerebrovasc Dis, 22:271278.

3. Milionis, H. (2015). Anemia on admission predicts short- and long-term outcomes in patients with acute ischemic stroke. Int J Stroke, 10:224-30.

4. Raphae, S., Katie, H. (2016). Impact of Hemoglobin Levels and Anemia on Mortality in Acute Stroke: Analysis of UK Regional Registry Data, Systematic Review and Meta-Analysis: Am Heart Assoc, 5(8):1-16.

5. Bhatia, R. (2004). Predictive value of routine hematological and biochemical parameters on 30day fatality in acute stroke, Neurol India; 52:220223.

6. Kellert, L., Martin, E. (2011). Cerebral oxygen transport failure? Stroke, 42:2832-2837.

7. Adams, H., Adams, J., Brott, T., Del Zoppo, G.J. (2003). Guidelines for the early management of patients with ischemic stroke. A scientific statement from the stroke council of the American Stroke Association. Stroke, 34:1056-1083.

8. Adams. H., Adams. R. (2005). Guidelines for the early management of patients with ischemic stroke. 2005 guidelines update .A scientific statement from the Stroke Council of the American Heart Association/American Stroke Association: Stroke, 36:916-921.

9. Felszeghy, K., Banisadr, G., \& Rostene, W. (2004). Dexamethasone downregulates chemokine receptor CXCR4 and exerts neuroprotection against hypoxi /ischemia-induced brain injury in neonatal rat: Neuroimmunomodulation, 11: 404-413.

10. Moro, A., Cardenas, A., Hurtado, O., Leza, J. (2004). Role of nitric oxide after brain ischaemia: Cell Calcium, 36: 265-275.

11. Shahar, A. (1991). Severe anemia associated with transient neurological deficits. Stroke, 22:12011202.

12. Hsiao,k.(2008). Severe anemia associated with transient ischemic attacks involving vertebrobasilar circulation. Am J Emerg Med, 26:e3-e4.

13. Nagel,T., Resnick, N. (1994) .Sheer stress selectivity upregulates intercellular adhesion molecule-1 expression in cultured human vascular endothelial cells. J Clin Invest, 94: 885-891.

14. Morigi, M., Zoja, C. (1995). Fluid shear stress modulates surface expression of adhesion molecules by endothelial cells. Blood, 85:16961703.

15. Ki, J., Kang, S. (2000). Bleeding and subsequent anemia: a precipitant for cerebral infarction. Eur Neurol, 43:201-208.

16. Kumar, M. (2009). Anemia and hematoma volume in acute intracerebral hemorrhage. Crit Care Med, 37:1442-1447.

17. Sheth, N., Gilson, A. (2011). Packed red blood cell transfusion and decreased mortality in intracerebral hemorrhage. Neurosurgery, 68:1286-1292.

18. Bhupesh, P., Suzanne, E., \& Judd. (2016). Hemoglobin Concentration and Risk of Incident Stroke in Community-Living Adults. Stroke, 47:2017-2024.

19. Gagnon, R., Zhang, J., \& Brand, N. (1994). Hematocrit and the risk of cardiovascular diseasethe Framingham study: a 34-year follow-up. Am Heart J, 127:674-682.

20. Zilong, H. (2013). A cohort study of patients with anemia on admission and fatality after acute ischemic stroke. Journal of Clinical Neuroscience, $20 ; 37-42$.

21. Tanne, D., Molshatzki, N. (2010). Anemia status, hemoglobin concentration and outcome after acute stroke: a cohort study. BMC Neuro, 10:22.

22. Singh, K., Szczech, L., \& Tang, L. (2006). Correction of anemia with epoetin alfa in chronic kidney disease. N Engl J Med, 355: 2085-2098. 